\title{
Prevalência de respiradores bucais em crianças de idade escolar
}

\author{
Prevalence of mouth breathing in children from an elementary school
}

Josiane $M$ arques Felcar ${ }^{1}$

Izabele Rafael Bueno ${ }^{2}$

Ana Carolina Silva M assan ${ }^{2}$

Roberta Pereira Torezan ${ }^{2}$

Jefferson Rosa Cardoso ${ }^{3}$

${ }^{1}$ Centro de Pesquisa em

CiênciasdaSaúde,

UniversidadeN ortedo

Paraná. Av. Paris 675, Jardim

Piza. 86041-140 Londrina

PR. josianefelcar@

sercomtel.com.br

${ }^{2}$ Graduada em Fisioterapia,

Universidade Nortedo

Paraná.

${ }^{3}$ Pós-graduação em Ciências

daReabilitação,

UniversidadeEstadual de

Londrina.
Abstract Theobjective of this article is to identify the prevalence of mouth breathing in children from an elementary school. 496 questionnaires were answered by 1st and 4th grade children's parents or sponsors in order to identify mouth-breathing. There were questions about habits, sleeping, behavior, eating, personal careand breathing. M annWhitney and the Chi-square tests were used to compare the variables between mouth-breathing and nose-breathing among the groups. To measure the exposure effect of the explanatory variables on mouse breathing, the test of logistic regression wasused and its magnitude was calculated through 0 dds Ratio. Thestatistical significance was set at $5 \%$, and the rate of returned questionnaires was $84.5 \%$. The prevalence of the mouthbreathing over this population was $56.8 \%$. The average age was 7 years old (6-9). There was no significant statistical difference between genders, considering $49.1 \%$ male and $50.9 \%$ female. The final model of logistic regression identified thevariables dribble, sleepswell (negativeassociation) and snores as factors that predict the occurrence of the mouth-breathing. The prevalence of mouthbreathing was similar to related in the literature. The variables dribble, sleeps well (negative association) and snores may be factors that predict the occurrence of mouth-breathing.

Key words M outh-breathing, Prevalence, Child, Pediatrics
Resumo Pretende-se identificar a prevalência de respiradores bucais em crianças de uma escola do ensino fundamental. Foram aplicados 496 questionáriosaos pais ou responsáveis das crianças de 1 à à 4a série de uma escola fundamental, para identificar respiradores bucais. 0 questionário incluía questões sobre hábitos, sono, comportamento, alimentação, cuidados pessoais e respiração. Para comparar as variáveis entre respiradores bucais e nasais, foi utilizado o teste de $M$ ann-Whitney e qui-quadrado. Para medir o efeito da exposição das variáveis explicativas sobre o desfecho primário, foi utilizada regressão logística esua magnitude foi calculada por meio do odds ratio. A significância estatística foi estipulada em 5\%. A taxa de devolução dos questionários foi de $84,5 \%$. A prevalência de respiração bucal nessa população foi $56,8 \%$. A mediana de idade foi sete anos (6-9). $N$ ão houve diferença estatisticamente significante entre os gêneros, $49,1 \%$ masculino e 50,9\% feminino. 0 modelo final de regressão logística identificou as variáveis baba, dorme bem (associação negativa) e ronca como fatores que predizem a ocorrência da respiração bucal. A prevalência de respiradores bucais foi semelhante à encontrada na literatura pesquisada. As variáveis babar, roncar e dormir bem (associação negativa) podem predizer a ocorrência da respiração bucal. Palavras-chave Respiração bucal, Prevalência, Criança, Pediatria 
Introdução

A respiração é uma função vital do organismo desenvolvida no primeiro momento de vida logo após o nascimento. Apenas as cavidades nasais possuem condições para filtrar partículas emicroorganismos do ar; assim, ele chega aos pulmões na temperatura ideal e favorece a oxigenação'.

A respiração nasal está associada a funções normais de mastigação, deglutição, postura da língua e lábios, além de proporcionar ação muscular correta que estimula o adequado crescimento facial e o desenvolvimento ósseo ${ }^{2}$.

A síndrome do respirador bucal, também conhecida como síndrome da face longa, é o conjunto desinais esintomas de quem respira parcial ou totalmentepela boca ${ }^{3}$. A respiração bucal apresenta etiologias multifatoriais, podendo ser de natureza obstrutiva como hipertrofia das tonsilas palatinas, hipertrofia das adenóides, desvio de septo nasal, pólipos nasais, alergias respiratórias, sinusites, hipertrofias de cornetos, posição de dormir, al eitamento artificial; ou decorrentes de hábitos bucais deletérios, tais como sucção digital ou de chupeta que, dependendo da intensidadee da frequência, deformam a arcada dentária e alteram todo o equilíbrio facial 4,5 . A criança que recebe 0 al eitamento materno natural e não por mamadeira, sobretudo nos primeiros meses de vida, tem maior possibilidade de ser um respirador predominantemente nasal durante a vida ${ }^{6}$.

0 respirador bucal apresenta uma série de alterações morfofuncionais, que se caracterizam pela boca aberta, hipotonia da língua e cianose intraorbitária7. 0 respirador bucal pode apresentar outras alterações importantes, tais como apnéia noturna, hipoventilação e desenvolvimento anormal do tórax ${ }^{3}$. Pode possuir características faciais como face al ongada e entristecida, lábios entreabertos e ressecados, bochechas caídas, língua hipotônica em posição inferior ou entreos dentes, ol heiras, flacidez detoda musculatura da face, má oclusão dentária, palato estreito e profundo $0^{8,9}$.

Geralmente, tem preferência por alimentos pastosos, come de boca aberta, costuma babar no travesseiro quando dorme e apresenta alterações no comportamento, como sono agitado, irritabilidade, dificuldade de concentração, inquieto, ansioso e impaciente. Pode também apresentar queda no rendimento escolar e baixa aptidão esportiva ${ }^{10}$

A respiração oral leva à protusão de cabeça, visando à manutenção da via respiratória pela necessidade de uma melhor respiração. Quando a cabeça se anterioriza, os ombros rodam internamente, deprimindo o tórax, o que leva a alterações no ritmo ena capacidade respiratória, pois o diafragma trabalha numa posição mais baixa e de forma assincrônica, o que ocasiona respiração rápida e curta e diminui a oxigenação ${ }^{11}$. Outras alterações musculoesqueléticas são hipercifose torácica e hiperlordose lombar, escoliose, escápulas aladas, depressão submamária, abdome proeminente, assimetrias pélvicas, hiperextensão de joelho, genu valgo, arco plantar desabado e hálux valgo ${ }^{12-16}$.

Devido à escassez de trabalhos relatando a prevalência de respiradores bucais, o presente trabalho tem como objetivo identificar a prevalência de respiradores bucais em crianças deuma escola do ensino fundamental da cidade de Londrina (PR).

\section{M etodologia}

Estudo transversal para a identificação de respiradores bucais em crianças de seis a onze anos. Foram aplicados 496 questionários aos pais ou responsáveis das crianças de primeira à quarta série do ensino fundamental de uma escola pública. 0 tempo de resposta foi de cinco dias. Diante dos questionários em mãos, foi dada uma segunda chance de mais três dias para os que não haviam respondido pudessem fazê-lo. Foi obtida previamente autorização da diretoria da escola para a aplicação dos mesmos. 0 trabalho foi aprovado pelo Comitê de Ética em Pesquisa da Universidade N orte do Paraná, Londrina, Paraná (CEP 273/05).

0 questionário foi elaborado de acordo com os critérios encontrados em indivíduos com respiração bucal ${ }^{3-11}$. Participaram da elaboração outros profissionais com experiência no assunto. Inicialmente, foi realizado um estudo piloto com trinta crianças no intuito de ajustar as questões finais. N este momento, foi realizado o teste da "água na boca" ${ }^{10,17}$ para confirmar se os respiradores bucais tinham de fato esta alteração. No questionário, foram incluídas questões relacionadas aos hábitos (aleitamento, chupar dedo ou chupeta), ao sono (posicionamento, ronco, sialorréia e qualidade do mesmo), ao comportamento ( concentração, atividadefísica), à alimentação (velocidade, quantidade, local, ingestão de líquidos durante as refeições, tempo e preferência alimentar), aos cuidados pessoais, se realizou algum tratamento (fisioterapia, fonoaudiologia, otorrino e ortodôntico), se rói unhas, usa ócu- 
los, toma alguma medicação e se já realizou alguma cirurgia.

Em relação à respiração, investigou-se por onde respira (boca, nariz ou ambos), intensidadeefrequência degripes, alergias, vacinas no caso de alguma alergia e respiração ruidosa.

Asvariáveis numéricas foram avaliadas quanto à distribuição denormalidade (teste de Shapiro-Wilk). Como as mesmas não atingiram seus pressupostos, estas foram apresentadas na forma de mediana e seus quartis (vide tabelas). As variáveis categóricas foram apresentadas por meio defrequência absoluta e relativa. Para comparar as variáveis numéricas entre os grupos, foi utilizado o teste de Mann-Whitney. Para avaliar a associação entre o desfecho primário e as variáveis categóricas, foi utilizada a análise univariada por meio do teste do qui-quadrado (com ou sem correção de Yates) ou teste exato de Fisher.

Para medir o efeito da exposição das variáveis explicativas sobre o desfecho primário (respiração bucal), foi utilizado o teste de regressão logística (método Stepwise) e sua magnitude foi calculada por meio do odds ratio (OR) com intervalo de confiança de $95 \%$. Neste procedimento, os resultados mais significantes (teste $F$ ) das associações das variáveis foram selecionados para o modelo inicial. Assim, foi verificado sea adição deuma determinada variável aumentaria a habi- lidadedo modelo em predizer o risco de ocorrência da respiração bucal. Caso a adição de uma variável aumentasse a predição, esta permanecia no modelo e, caso contrário, era rejeitada. A significância estatística foi estipulada em $5 \%(p \leq 0,05)$. Para a análise dos dados, foi usado o programa Statistical Package for the Social Sciences (SPSS) versão 13.0 para Windows.

\section{Resultados}

A taxa de devolução dos questionários foi de $84,5 \%$. A prevalência de respiração bucal nessa população foi de $56,8 \%$.

A mediana da idade entre os respiradores bucais (RB) foi de sete anos (6-9) de idade e entre os respiradores nasais (RN) foi de oito anos (6-9) de idade $(p<0,001)$. Dos RB, $114(49,1 \%)$ eram do gênero masculino e 118 (50,9\%), do gênero feminino. D os RN , 77 (43,8\%) eram do gênero masculino e $99(56,3 \%)$, do gênero feminino $(p=0,280)$.

$\mathrm{Na}$ Tabela 1, encontram-se os dados relativos aos hábitos que podem intervir no tipo respiratório.

Todas as variáveis relacionadas ao sono foram estatisticamente significantes e encontramse na Tabela 2, juntamente com os dados relativos ao comportamento das crianças.

Tabela 1. Resultados relacionados às variáveis dos hábitos das crianças respiradoras bucais e nasais.

\begin{tabular}{|c|c|c|c|}
\hline Hábitos & Respirador bucal & Respirador nasal & $P$ \\
\hline Aleitamento materno & & & 0,978 \\
\hline $\operatorname{Sim}(n ; \%)$ & $212(92,6)$ & $161(92,0)$ & \\
\hline Não $(n ; \%)$ & $17(7,4)$ & $14(8,0)$ & \\
\hline Tempo al eitamento materno & & & $<0,001$ \\
\hline$(\mathrm{Md} ; 10-30 \mathrm{q})$ em anos & $0,75(0,33-1)$ & $0,71(0,41-2)$ & \\
\hline Uso de mamadeira & & & 0,573 \\
\hline $\operatorname{Sim}(n ; \%)$ & $189(81,8)$ & $140(79,1)$ & \\
\hline Não $(n ; \%)$ & $42(18,2)$ & $37(20,9)$ & \\
\hline Tempo de mamadeira & & & $<0,001$ \\
\hline$(\mathrm{Md} ; 10-30 \mathrm{q})$ em anos & $4(1-5)$ & $3(2-5)$ & \\
\hline Uso de chupeta & & & 0,704 \\
\hline $\operatorname{Sim}(n ; \%)$ & $106(45,9)$ & $77(43,5)$ & \\
\hline Não $(n ; \%)$ & $125(54,1)$ & $100(56,5)$ & \\
\hline Tempo de chupeta & & & $<0,001$ \\
\hline$(\mathrm{Md} ; 10-30 \mathrm{q})$ em anos & $0(0-3)$ & $0(0-3)$ & \\
\hline Chupar o dedo & & & 0,439 \\
\hline $\operatorname{Sim}(n ; \%)$ & $26(11,3)$ & $15(8,5)$ & \\
\hline Não $(n ; \%)$ & $204(88,7)$ & $162(91,5)$ & \\
\hline Tempo de dedo & & & $<0,001$ \\
\hline$\left(\mathrm{Md} ; 10-3^{\circ} \mathrm{q}\right)$ em anos & $0(0-0)$ & $0(0-0)$ & \\
\hline
\end{tabular}


Tanto nas crianças com respiração bucal quanto nas com respiração nasal, a mediana do tempo que levam para comer foi de 15 (10-20) minutos $(p<0,001)$. Os outros dados da alimentação estão apresentados na Tabela 3.

Das crianças que apresentavam respiração bucal, 34 (14,9\%) fizeram tratamento fisioterápico, quinze $(6,7 \%)$, tratamento fonoaudiológico, seis $(2,8 \%)$, tratamento ortodôntico e $56(25,2 \%)$, tratamento com otorrinolaringologista. E das com respiração nasal, dezoito (10,3\%) fizeram fisioterapia, nove $(5,2 \%)$, fonoaudiologia, dez $(6,3 \%)$, tratamento ortodôntico e dezoito (10,5\%), tratamento com otorrinolaringologista.

Os resultados estatisticamente significativos das associações das variáveis da análise univaria- da foram selecionados para o modelo usado na análise multivariada (Tabela 4).

Participaram desse modelo as seguintes variáveis: dorme bem, ronca, baba, acorda à noite, sono agitado, atividade física (cansa fácil), como come, quanto comee onde come. 0 model o final de regressão logística identificou as variáveis baba, qualidadedo sono eronco como fatores quepredizem a ocorrência da respiração bucal. As crianças que babam têm 1,93 de chance a mais de apresentar ser RB, IC 95\% [1,14; 3,26] e as que roncam têm 3,49 de chance a mais de ser também RB, IC 95\% [1,89; 6,44]. Já dormir bem esteve associada negativamente à respiração bucal com OR de 0,75 , IC 95\% $[0,01 ; 0,57]$.

Tabela 2. Resultados relacionados às variáveis do sono e comportamento das crianças respiradoras bucais e nasais.

\begin{tabular}{|c|c|c|c|}
\hline & Respirador bucal (n; \%) & Respirador nasal (n; \%) & $P$ \\
\hline \multicolumn{4}{|l|}{ Sono } \\
\hline Dorme bem & & & $<0,001$ \\
\hline Sim & $203(87,5 \%)$ & $175(98,9 \%)$ & \\
\hline Não & $29(12,5 \%)$ & $2(1,1 \%)$ & \\
\hline Ronca & & & $<0,001$ \\
\hline Sim & $88(38,4 \%)$ & $21(12,1 \%)$ & \\
\hline Não & $141(61,6 \%)$ & $152(87,9 \%)$ & \\
\hline Baba & & & $<0,001$ \\
\hline Sim & $104(45,2 \%)$ & $37(21,1 \%)$ & \\
\hline Não & $126(54,8 \%)$ & $138(78,9 \%)$ & \\
\hline Acorda à noite & & & 0,001 \\
\hline Sim & $83(36,2 \%)$ & $37(20,9 \%)$ & \\
\hline Não & $146(63,8 \%)$ & $140(79,1 \%)$ & \\
\hline Sono agitado & & & $<0,001$ \\
\hline Sim & $94(40,5 \%)$ & $38(21,7 \%)$ & \\
\hline Não & $138(59,5 \%)$ & $137(78,3 \%)$ & \\
\hline Concentração & & & 0,378 \\
\hline Dispersa & $40(18,3)$ & $16(10,0)$ & \\
\hline Atenta & $92(42,2)$ & $93(58,1)$ & \\
\hline Hiperativa & $25(11,5)$ & $16(10,0)$ & \\
\hline Agressiva & $1(0,5) 23$ & $0(0,0)$ & \\
\hline Irritada & $(10,6)$ & $15(9,4)$ & \\
\hline Outras & $37(17,0)$ & $20(12,5)$ & \\
\hline \multicolumn{4}{|l|}{ Atividade física } \\
\hline Gosta & & & 0,659 \\
\hline Sim & $217(95,2)$ & $169(96,6)$ & \\
\hline Não & $11(4,8)$ & $6(3,4)$ & \\
\hline Realiza & & & 0,129 \\
\hline Sim & $138(66)$ & $119(73,9)$ & \\
\hline Não & $71(34)$ & $42(26,1)$ & \\
\hline Cansa fácil & & & 0,002 \\
\hline Sim & $71(32,6)$ & 29 (18) & \\
\hline Não & $147(67,4)$ & $132(82)$ & \\
\hline
\end{tabular}


Tabela 3. D ados referentes à alimentação das crianças respiradoras bucais e nasais.

\begin{tabular}{|c|c|c|c|}
\hline Alimentação & Respirador bucal ( $\mathrm{n} ; \%)$ & Respirador nasal (n; \%) & $P$ \\
\hline \multicolumn{3}{|l|}{ Como come } & 0,023 \\
\hline Rápido & $77(33,2)$ & $40(22,9)$ & \\
\hline D evagar & $155(66,8)$ & $135(77,1)$ & \\
\hline \multicolumn{3}{|l|}{ Quanto come } & 0,031 \\
\hline Pouco & $79(33,9)$ & $39(22,4)$ & \\
\hline M édio & $130(55,8)$ & $118(67,8)$ & \\
\hline M uito & $24(10,3)$ & $17(9,8)$ & \\
\hline \multicolumn{3}{|l|}{ Onde come } & 0,045 \\
\hline Frente TV & $42(18,1)$ & $44(25,3)$ & \\
\hline Sala & $66(28,4)$ & $30(17,2)$ & \\
\hline Cozinha & $112(48,3)$ & $91(52,3)$ & \\
\hline M ais de um local & $12(5,2)$ & $9(5,2)$ & \\
\hline \multicolumn{3}{|l|}{ Comida preferida } & 0,223 \\
\hline Amassada & $9(4,0)$ & $12(7,1)$ & \\
\hline Triturada & $7(3,1)$ & $5(2,9)$ & \\
\hline Papa & $3(1,3)$ & $5(2,9)$ & \\
\hline Sólida & $149(66,2)$ & $117(68,8)$ & \\
\hline Sopa & $32(14,2)$ & $22(12,9)$ & \\
\hline Sólida e sopa & $25(11,1)$ & $9(5,3)$ & \\
\hline \multicolumn{3}{|l|}{ Engasga } & 0,231 \\
\hline Sim & $17(7,5)$ & $9(5,2)$ & \\
\hline Não & $209(92,5)$ & $165(94,8)$ & \\
\hline \multicolumn{3}{|c|}{ Líquidos durante as refeições } & 0,171 \\
\hline Sim & $174(76)$ & $140(80,5)$ & \\
\hline Não & $55(24)$ & $34(19,5)$ & \\
\hline
\end{tabular}

Tabela 4. Análise multivariada das três principais variáveis relacionadas com respiração bucal.

\begin{tabular}{lccrrr}
\hline \multicolumn{1}{c}{ Variável } & Estimativa & Erro padrão & $\chi^{2}$ & $P$ & Odds ratio [IC 95\%] \\
\hline Intercept & 2,31 & 1,03 & 4,97 & 0,026 & \\
Ronca & 1,25 & 0,31 & 16,08 & $<0,001$ & $3,49[1,89 ; 6,44]$ \\
Dorme bem & $-2,58$ & 1,03 & 6,21 & 0,013 & $0,75[0,01 ; 0,57]$ \\
Baba & 0,66 & 0,26 & 6,10 & 0,013 & $1,93[1,14 ; 3,26]$ \\
\hline
\end{tabular}

\section{Discussão}

A literatura é escassa em relação à prevalência de RB em crianças; estudo realizado em Recife (PE) com este fim mostrou uma prevalência de $53,3 \%{ }^{17}$, que foi semelhante à encontrada em nosso estudo. Um trabalho venezuelano encontrou uma prevalência um pouco maior de $63 \%{ }^{18}$. Estas diferenças talvez possam ser justificadas pelos critérios de diagnóstico e as diferentes metodologias usadas nos estudos. No presente tra- balho, foram incluídas crianças com respiração oral e mista no mesmo grupo (RB), conforme preconizado pela literatura ${ }^{19}$.

Neste estudo, houve um leve predomínio de respiração bucal no sexo feminino, sem significância estatística, dado que discorda dos demais autores, nos quais o predomínio de RB foi no sexo masculino ${ }^{17,18,20}$. O predomínio sobre um gênero ou outro foi discreto tanto em nosso estudo como nos citadosacima, demonstrando que estes dados não parecem ser relevantes. 
Embora a maioria dos autores concorde com a definição do respirador bucal, não há unanimidade quanto ao método mais preciso de identificá-lo. 0 relato dos pais ou responsáveis com re lação à presença de roncos, postura de boca aberta, obstrução nasal e salivação excessiva devesempre ser valorizado pelo profissional de saúde ${ }^{21}$.

0 ar que entra pela boca não é umidificado, aquecido e filtrado, criando uma porta de entrada para agentes agressores, pois não há barreiras imunológicas naturais contra eles, causando maior frequência de infecções das vias aéreas superiores ${ }^{3,7}$. Os RB costumam apresentar rinites, sinusites, amidalites, bronquites e pneumonias, além de otites ${ }^{10}$.

A amamentação possui reconhecidos ben efíciosnutricionais, imunológicos, cognitivos, econômicos e sociais, devendo ser oferecida como forma exclusiva de alimentação do lactente atéo sexto mês de vida ${ }^{22}$. 0 padrão correto de respiração pode ser prejudicado pelo desmame precoce (antes dos seis meses de vida). Durante a amamentação, o lactante mantém a postura de re pouso dos lábios ocluídos e a respiração nasal. Com o desmame precoce, a postura de lábios entreabertos torna-se mais comum, favorecendo a respiração oral 6 . 0 desmame precoce é um fato que poderia explicar a alta incidência de RB em crianças amamentadas deste estudo. Além disso, a amamentação previne infecções respiratórias, graças aos componentes presentes no leite materno ${ }^{23}$.

A utilização de mamadeiras, mesmo em associação com aleitamento materno, leva a alterações no desenvolvimento orofacial ${ }^{24}$. Com 0 desmame precoce, a criança não supre suas necessidades de sucção e acaba adquirindo hábitos de sucção não nutritiva ${ }^{23}$.

Os hábitos orais de sucção viciosos mais frequentes são dedo, lábios, bochecha e objetos como chupeta e mamadeira que podem provocar alterações na arcada dentária e na mordida, hipotonia de órgãos fonoarticulatórios, respiração bucal, onicofagia e outros ${ }^{25}$. Em N ova Deli, na Índia, 25,5\% das crianças em idade escolar apresentam algum hábito oral, sendo o mais frequente o movimento de empurrar a língua ${ }^{26}$. Em nosso estudo, mais da metade dos RB analisados utilizaram chupeta e poucos possuíram 0 hábito da sucção digital; porém, a grande maioria fez uso de mamadeira, sendo que o tempo de utilização dos três tevesignificânciaestatística com relação à intervenção no tipo respiratório.

Observou-se, neste estudo, que os RB analisados referem dormir bem; porém, a incidência de ronco, baba enquanto dorme, sono agitado e acordar à noite foi significativamente maior nos RB em relação aos nasais. Os distúrbios obstrutivos do sono são relativamente frequentes na população pediátrica e incluem o ronco e a apnéia obstrutiva do sono, sendo uma das causas mais comuns a obstrução de vias aéreas superiores e consequente respiração bucal ${ }^{27-29}$.

A resistência das vias aéreas superiores durante o sono é significativamente menor na respiração nasal do que na respiração bucal. Além de aumentar a resistência das vias aéreas superiores, a via oral é associada ao agravamento da apnéia obstrutiva do sono. Esta diferença de resistência entre via nasal eoral faz com queindivíduos saudáveis tenham uma respiração predominantementenasal ${ }^{30}$. Entretanto, nos RB, muitas vezes a obstrução das vias aéreas superiores não permite uma respiração nasal e consequentemente uma boa noite de sono.

O sono é agitado, de má qualidade e com pesadel os frequentes, o que leva à hipersonolência diurna, cansaço, ansiedade, desânimo, falta concentração e aten ção ${ }^{31}$, à diminuição do rendimento escolar ${ }^{10,27}$ e até à depressão. Para que a língua não gere ainda mais dificuldades respiratórias, o respirador bucal tenta encontrar a posição mais confortável para dormir, geralmenteem decúbito ventral ou lateral ${ }^{3}$.

A respiração bucal resulta em posição alterada da língua e lábios entreabertos; as alterações no tônus da musculatura facial levam a uma hipotonia, dificultando a manutenção da boca fechada, mesmo após solucionar os problemas obstrutivos. A mastigação é ineficiente e leva a problemas digestivos e engasgos pela incoordenação da respiração com a mastigação, deglutição atípica com ruído, projeção anterior de língua, contração exagerada de orbicular, movimentos compensatórios de cabeça, fala imprecisa, trancada, com excesso de saliva, sem sonorização pelas otites frequentes, com alto índice de ceceio anterior ou lateral e voz rouca ou anasalada. Eles esmagam o alimento contra o palato com o dorso da língua, o quetorna a mastigação mais vertical esem lateralização demandíbula. A deglutição real iza-secom pressão atípica do dorso da língua e com participação da musculatura perioral, háanteriorização delíngua, emovimentos associados de cabeça ${ }^{32}$.

Em repouso, $80 \%$ das pessoas saudáveis respiram exclusivamente pelo nariz. Duranteo exercício, há um aumento da ventilação e uma mudança na via respiratória que vai de nasal para oronasal; então, a maior parte da ventilação é 
realizada pela boca ${ }^{33}$. 0 respirador bucal fica sem esta opção.

Uma série de fatores já citados, tais como alteração do sono, capacidade pulmonar diminuída, alimentação inadequada, entre outros, leva o respirador bucal a ter baixa aptidão esportiva ${ }^{10,31}$. Foi observado que a maioria das crianças com respiração bucal gosta e realiza al guma atividade física, embora alguns apresentem cansaço excessivo durantea realização detais atividades, provavel mente em decorrência da respiração inadequada.

As crianças com respiração bucal apresentam diversas alterações que, se não diagnosticadas precocemente, podem trazer consequências inesperadas, tanto no comportamento, no psicológico, no físico e, portanto, no relacionamento pessoal ${ }^{17}$. Assim sendo, é de extrema importância a implantação de políticas de saúde, com o intuito deincentivar hábitos que possam evitar a respiração bucal, como a amamentação, e coibir hábitos que possam levar a desenvolvêla. Bem como diagnosticar precocemente, de forma a melhorar a qualidade de vida de crianças portadoras de respiração bucal.

A respiração bucal não éuma alteração fisiológica e sim patológica, que pode provocar alterações funcionais e morfológicas em todo organismo. 0 diagnóstico, prevenção e tratamento devem ser integrados com áreas da fisioterapia, fonoaudiologia, ortodontia, otorrinolaringologia e, às vezes, psicologia e nutrição para al cançar resultados satisfatórios, ou seja, é muito importante 0 tratamento multidisciplinar, $3,10,15,17,31-36$.

Conclui-se que a prevalência de RB foi semeIhante à encontrada na literatura pesquisada. As variáveis babar, roncar e dormir bem (associação negativa) podem predizer a ocorrência da respiração bucal.

\section{Colaboradores}

JM Felcar participou do planejamento, coleta de dados, metodologia, análise, interpretação e discussão dos resultados. IR Bueno, ACS M assan e RP Torezan participaram do planejamento, coleta de dados e discussão dos resultados. JR Cardoso participou da metodologia, análise e interpretação dos resultados. Todos colaboraram com a elaboração do texto.

\section{Referências}

1. Spinelli M LM , Casanova PC. Respiração bucal [texto na Internet]. [acessado 2007 nov 08]. Disponível em: http://www.odontologia.com.br/artigos.asp?id= 224\&idesp\%2B14\&ler=s

2. Faria PMT, Ruellas ACO, M atsumoto MAN, Anselmo-Lima WT, Pereira FC. Dentofacial morphology of mouth breathing children. Braz Dent J. 2002; 13(2):129-132.

3. M iranda PPC, M ashuda SYK, Periotto MC, Araújo $\mathrm{RJH}$. Enfoque multidisciplinar na síndrome do respirador bucal. Rev Paulista de Odontol. 2002; 24(3):4-8.

4. Fransson JMD, Magnani MBBA, Nouer DF, Siqueira VCV, Lunardi N. Estudo cefalométrico comparativo entre respiradores nasais e predominantemente bucais. Rev Bras Otorrinolagol. 2006; 72(1):72-82.

5. Lopatiené K, Babarskas A. Malocclusion and upper airway obstruction. M edicina 2002; 38(3):277-283.

6. Neiva FCB, Cattoni DM, Ramos JLA, Issler H. Desmame precoce: implicações para o desenvolvimento motor-oral. J. Pediatr. 2003; 79(1):7-12.

7. Yi LC, Guedes ZCF, Pignatari S, Weckx LLM. Avaliação postural em crianças de 5 a 12 anos que apresentam respiração oral. Fisioterapia em movimento 2003;16(3):29-33.

8. Cintra CFSC, Castro FFM, Cintra PPVC. As alterações oro-faciais apresentadas em pacientes respiradores bucais. Rev Bras Alerg Imunopatol. 2000; 23(2):78-83.

9. Rodrigues MEM, Rodrigues PCM SM. Respiração bucal: diagnóstico e tratamento bucal [texto na Internet]. [acessado 2007 jul 20]. Disponível em: http:/ /www.abom.org.br/texto_artigo02.htm 
10. Lusvarghi L. Identificando o respirador bucal. Rev APCD. 1999; 53(4):265-274.

11. Quintão FC, Andrade DC, Lagôa LC. A síndrome do respirador oral, suas influências na postura e a atuação da fisioterapia [texto na Internet]. [acessado 2007 jul 29]. Disponível em: http://www.wgate. com.br/fisioweb

12. Krakauer LH. Guilherme A. Relação entre respiração bucal e alterações posturais em crianças: uma análise descritiva. R Dental Press Ortodon Ortop Facial 2000; 5(5):85-92.

13. Godoy P, Niitsuma LEM, Caromano FA. Avaliação funcional fisioterapêutica do respirador bucal. Arq Ciênc Saúde Unipar 2000; 4(2):111-120.

14. Silva FB, Sampaio LM M, Carrascosa AC. Avaliação fisioterapêutica dos sistemas mastigatório e respiratório de um portador de síndrome otodental: um estudo de caso. Rev Bras Fisioter. 2006; 10(1):133-136.

15. M arins RS. Síndrome do respirador bucal e modificação posturais em crianças e adolescentes: a importância da fisioterapia na equipe interdisciplinar. Fisioterapia em M ovimento 2001; 14(1):45-52.

16. Lima LCO, Baraúna MA, Sologurem MJJ, Canto RST, Gastaldi AC. Postural alterations in children with mouth breathing assessed by computerized biophotogrammetry. J Appl Oral Sci. 2004; 12(3):232-237.

17. M enezes VA, Leal RB, Pessoa RS, Pontes RMES. Prevalência e fatores associados à respiração oral em escolares participantes do projeto Santo Amaro-Recife, 2005. Rev Bras Otorrinolaringol. 2006; 72(3):394-399.

18. Parra Y. El paciente respirador bucal una propuesta para el Estado Nueva Esparta 1996 - 2001. Acta Odontol Venez. 2004; 42(2):97-106.

19. Lara $A M A E$, Silva M FC. Respiração bucal: revisão da literatura. Virtual Odontol. 2007; 1(4):28-32.

20. Di Francesco RC, Passerotii G, Paulucci B, Miniti A. Respiração oral na criança: repercussões diferentes de acordo com o diagnóstico. Rev Bras Otorrinolaringol. 2004; 70(5):665-670.

21. Barros JRC, Becker HM G, Pinto JA. Avaliação de atopia em crianças respiradoras bucais atendidas em centro de referência. J. Pediatr. 2006; 82(6):458-464.

22. Chaves RG, Lamounier JA, César CC. Fatores associados com a duração do aleitamento materno. J. Pediatr. 2007; 83(3):241-246.

23. Voi Trawitzki LV, Anselmo-Lima WT, Melchior MO, Grechi TH, Valera FCP. Aleitamento e hábitos orais deletérios em respiradores orais e nasais. Rev Bras Otorrinolaringol. 2005; 71(6):747-751.

24. Carrascoza KC, Possobon RF, Tomita LM, Moraes $A B A$. Consequências do uso da mamadeira para 0 desenvolvimento orofacial em crianças inicialmente amamentadas ao peito. J. Pediatr. 2006; 82(5):395397.
25. Cavassani VGS, Ribeiro SG, Nemr NK, Greco AM Köhle J, Lehn CN. Hábitos orais de sucção: estudo piloto em população de baixa renda. Rev Bras Otorrinolaringol. 2003; 69(1):106-110.

26. Kharbanda OP, Sidhu SS, Sundaram KR, Shukla DK. Oral habits in school going children of Delhi: a prevalence study. J Indian Soc Pedo Prev Dent. 2003; 21(3):120-124.

27. Ng DK, Kwok K, Cheung JM, Leung S, Chow $P$, Wong WH, Chan $\mathrm{CH}, \mathrm{Ho}$ JC. Prevalence of sleep problems in Hong Kong primary school children: a community-based telephone survey. Chest 2005; 128:1315-1323.

28. Uema SFH, Vidal MVR, Fujita R, Moreira G, Pignatari SSN. Avaliação comportamental em crianças com distúrbios obstrutivos do sono. Rev Bras Otorrinolaringol. 2006; 72(1):120-123.

29. Urschitz MS, Guenther A, Eitner S, Urschitz-Duprat PM, Schlaud M, Ipsiroglu OS, Poets CF. Risk Factors and Natural History of $\mathrm{H}$ abitual Snoring. Chest 2004; 126:790-800.

30. Fitzpatrick M F, M CLean $H$, Urton AM, Tan A, O'Donnell D, Driver HS. Effect of nasal or oral breathing route on upper airway resistance during sleep. Eur Respir J. 2003; 22:827-832.

31. Carvalho, M. P. Respiração bucal: uma visão fonoaudiológica na atuação multidisciplinar. Revista de Otorrinolaringologia 2000; 7(2):54-59.

32. Frejman MW. Respiração bucal [texto na Internet]. [acessado 2007 jul 20]. Disponível em: http:// www.fonoaudiologia.com/trabalhos/artigos/artigo018/artigo-018-respira.htm

33. Amis TC, O'N eill N, Wheatley JR. Oral airway flow dynamics in healthy humans. J Physiol. 1999; 515:293-298.

34. Martins AS, Vieira M M, Vieira M R Pereira PKS. Estudo da relação entre respiração oral e perda auditiva. Rev Soc Bras Fonoaudiol. 2006; 11(3):175-180.

35. Ribeiro EC, Soares LM. Avaliação espirométrica de crianças portadoras de respiração bucal antes e após intervenção fisioterapêutica. Fisioterapia Brasil 2003; 4(3):163-167.

36. Vasconcellos IC, Gosling FB. Avaliação funcional do paciente respirador bucal. RBO 2003; 60(5):321323.

Artigo apresentado em 19/11/2007

Aprovado em 27/07/2008

Versão final apresentada em 28/07/2008 